\title{
Designing the care environment in hospitals and nursing homes: a pilot for a new and reliable method for determining the optimum space to live in and work in
}

\author{
Knibbe, J.J. ${ }^{*}$, E.Waaijer ${ }^{2}$ \\ LOCOmotion, Research in Health Care, Brinkerpad 29, 6721 WJ Bennekom, The Netherlands.
}

\begin{abstract}
One of the major problems encountered in health care during the process of implementing ergonomic changes is the lack of space. There is often not enough space to work with larger equipment like patient lifters and shower chairs or other medical devices necessary in a preventive program. This leads to undesirable extra physical load for nurses. Therefore it is imperative that in the process of designing the health care environment ergonomic considerations are taken into account. A method to assess the optimum size and shape of the space required is developed and the value of the method is tested in a pilot setting. The pilot is currently underway and the results will be presented on the final poster.
\end{abstract}

Keywords: nurses; architecture; building

\section{Introduction}

One of the major problems encountered in health care during the process of implementing ergonomic changes is the lack of space. There is often not enough space to work with larger equipment like patient lifters and shower chairs or other medical devices. This leads to undesirable extra effort and physical load for nurses instead of less physical load.

Therefore it is imperative that in the process of designing the health care environment ergonomic considerations are taken into account. However, it is difficult to find reliable measurements of the space needed and to find clear criteria for determining sufficient space. There are some examples in the literature, but they are often more related to clinical care and less to the nursing home, care home and home care environment.
Furthermore the examples given in the literature are not well validated or it is not clear how they were validated. That means that nurse managers and architects are not convinced of the need for space. They often consider the dimensions given as being too large and not really necessary.

Therefore there is a strong need to clarify why indeed there is such a great need for space for nurses to work in. Previous experience by the first author suggests directions for new methods and they are currently being piloted.

\section{Method: Internetapplication to design the health care environment}

An internet application was developed that helps nurses and architects to choose activities and the

*Corresponding author. E-mail: j.j.knibbe@planet.nl.

${ }^{11}$ LOCOmotion, www.locomotion.nu

${ }^{2}$ Arjohuntleigh, elly.waaijer@arjohuntleigh.com

1051-9815/12/\$27.50 @ 2012 - IOS Press and the authors. All rights reserved 
space demands that is associated with these activities for a whole range of activities (ww.zorginwoningen.nl). It was an award winning project and we participated actively in this project. Nevertheless new products constantly appear on the market and there was also a strong need to be able to determine the spatial requirements (sizes and shapes) in a more reliable, more valid and also a more practical way.

Therefore a pilot study was undertaken that assesses under real life conditions the space requirements with the use of modern video-analyses.

\section{Results}

In this new study 5 nurses performed 5 different activities with 5 patients of increasing level of functional mobility. There movements and use of space was assessed by means of video from a height of 8 meters. These videos are currently being analyzed by means of SIMI video and motion analyses.

\section{Conclusions}

The differences between the nurses, between the activities and between de degrees of mobility will be presented and a final conclusion will be drawn as to the validity, the reliability and the practicality of this method for standardized use to determine space requirements in the health care sector for nurses working according to ergonomic guidelines.

As the study is still underway, the poster will give in depth and visually illustrated insight in the methods used and the results.

\section{References}

